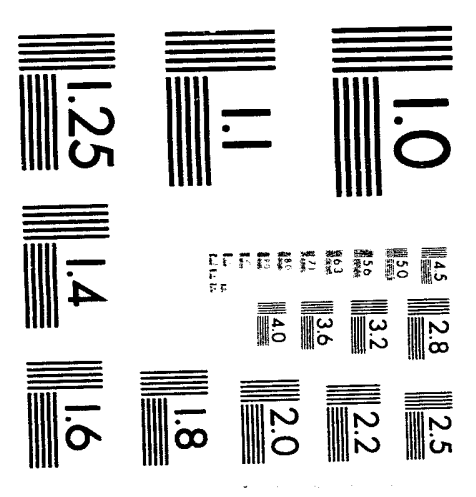



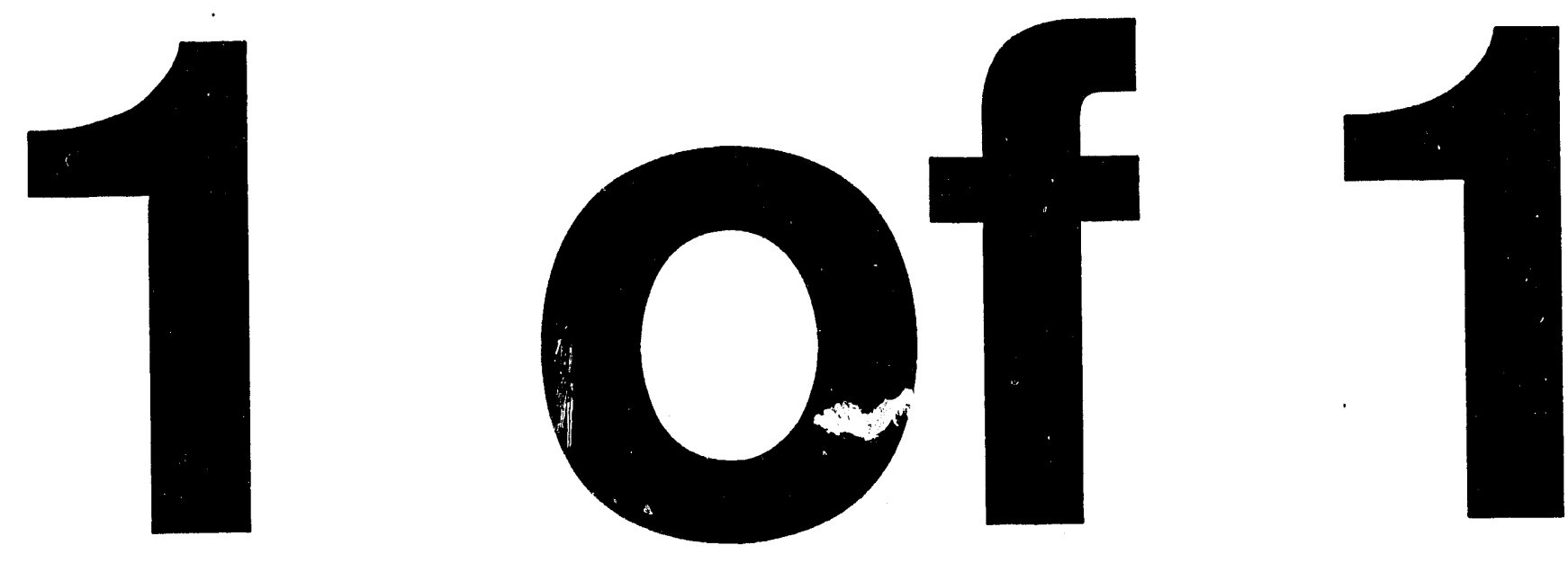
4.93950

UCRL-ID-114812

\section{Data Compression Enhancements to the Distributed Interactive Simulation Protocol for Use by Entity-Level Simulations}

\section{Edward T. Powell}

June 24, 1993

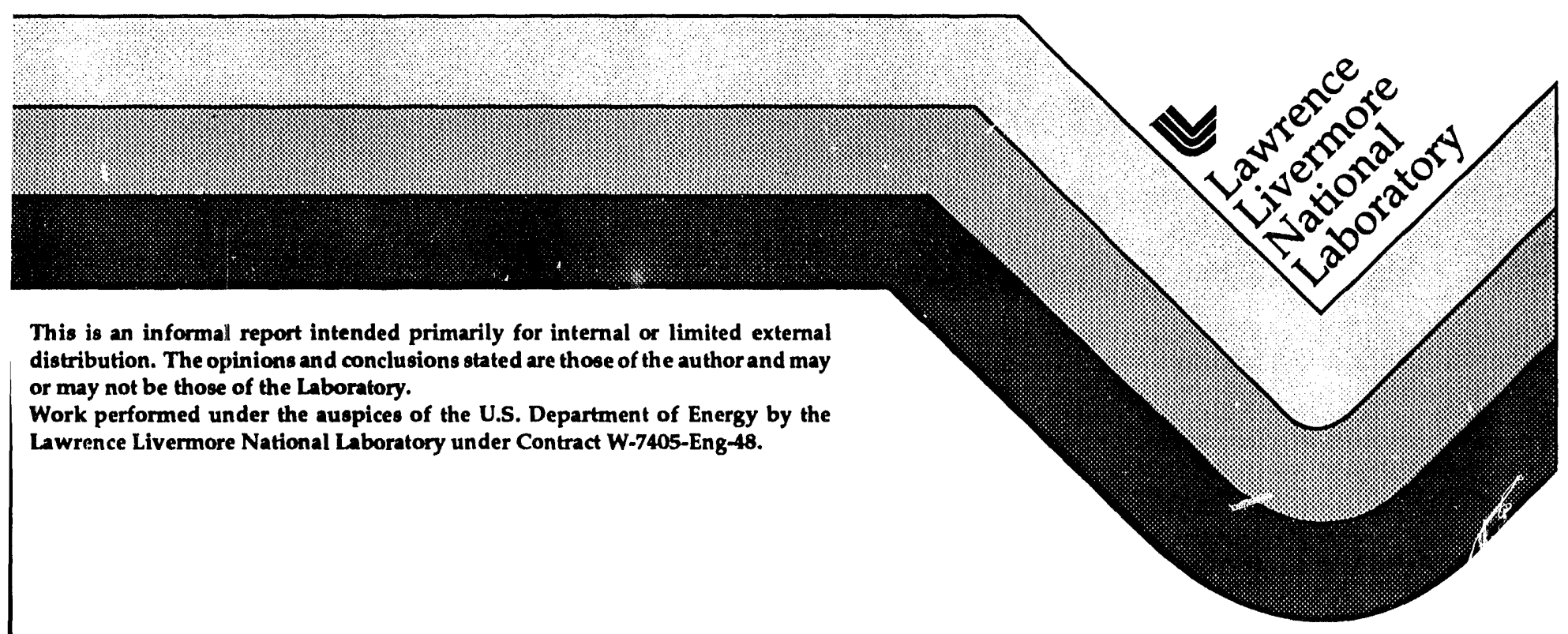

This is an informal report intended primarily for internal or limited external distribution. The opinions and conclusions stated are those of the author and may may not be those of the Laboratory.

Work performed under the auspices of the U.S. Department of Energy by the awrence Livermore National Laboratory under Contract W-7405-Eng-48. 


\section{DISCLAIMER}

This document was prepared as an account of work sponsored by an agency of the United States Government. Neither the United States Government nor the University of California nor any of their employees, makes any warranty, express or implied, or assumes any legal liability or responsibility for the accuracy, completeness, or usefulness of any information, apparatus, product, or process disclosed, or represents that its use would not infringe privately owned rights. Reference herein to any specific commercial products, process, or service by trade name, trademark, manufacturer, or otherwise, does not necessarily constitute or imply its endorsement, recommendation, or favoring by the United States Government or the University of California. The views and opinions of authors expressed herein do not necessarily state or reflect those of the United States Government or the University of California, and shall not be used for advertising or product endorsement purposes.

This report has been reproduced directly from the best available copy.

$A$ vailable to DOE and DOE contractors from the Omice of Scientific and Technical Information

P.O. Box 62, Oak Ridge, TN 37831

Prices a vailable from (615) 576.8401, FTS 626.8401

A vailable to the public from the

National Technical Information Service

US. Department of Commerce

5285 Port Royal Rd.,

Springfield, VA 22161 


\begin{abstract}
A series of new Protocol Data Units (PDUs) that send only limited entity-state information over a Distributed Interactive Simulation (DIS) network is described. These PDUs are tailored specifically to simulations that model numerous entities rather than simulators modeling a single entity. The use of these new PDUs could (under certain circumstances) realize compression factors of 16,32 , or up to 1200 to one in the amount of data that needs to be sent over a DIS network.
\end{abstract}

\title{
DEFINITIONS
}

Entity: A single military item on the battlefield, such as a tank, a truck, an aircraft, a helicopter, a dismounted soldier, etc. Anything which fights or moves as a single item.

Unit: A collection of units and entities, such as a section, platoon, company, battalion, etc. Units are hierarchically defined. Any group of entities that fight together with a common purpose.

Simulation: A computer program that models the actions of one or more entities or units on the battlefield. Examples include Janus, JCM, UCCATS, VIC, CBS, CASTFOREM, etc. Simulations can be interactive (having player input) or noninteractive (scripted or intelligent) or be a combination of both.

Simulator: An interactive system that models one entity on the battlefield in a manner such that the operator (player) can reasonably be said to take the same actions as he would in the corresponding platform on a real battlefield. Examples include an aircraft simulator, a tank simulator (SIMNET), etc.

PDU: Protocol Data Unit. A packet of information, defined by a recognized standard, that is designed to allow the transmission of the information over a computer network. There can be many different types of PDUs defined, representing many different types of information.

Entity State PDU (ESPDU): A PDU that describes an entity's appearance, position, velocity, etc. Issued when an entity's dead reckoning model of itself is sufficiently different from its high-resolution model of itself.

\section{INTRODUCTION}

The Distributed Interactive Simulation (DIS) uses a technique called Rernote Entity Approximation (REA), sometimes called Dead Reckoning, to reduce the amount of network traffic generated by any one simulator. During an exercise, each simulation node on the network maintains a list of known battle-

field entities. This list may be exhaustive and contain every entity in the entire 
exercise, or it may be limited to only those entities that are within range of the node's own entities' sensors or weapons. Each node maintains a dead reckoning model of each entity on its list, including both the entities controlled by other nodes (called othership entities) and entities controlled by that particular node (called ownship entities). Each node, of course, also maintains a high-resolution movement model of its ownship entities. When the position and/or orientation of an ownship entity given by the high resolution model differs from that obtained using the dead reckoning model by a given threshold, an Entity State Protocol Data Unit (shown in Appendix B) is issued by the node onto the network. The node then updates its dead reckoning model using this new information. This REA technique, while very effective for the interaction of simulators, does not go far enough to reduce the network load when entity-level simulations are interacting.

This paper presents ideas for data compression specifically designed for simulations interacting over a distributed network. It presents new PDUs which would carry the compressed information. The primary attribute of simulations being exploited for compression purposes is that simulations model man $y$ entities at once. Therefore it is likely that they would need to issue large numbers of ESPDUs for their entities. While it is envisioned that simulators could be part of an exercise with these simulations and could read and understand these new PDUs, the compression scheme relies on simulations needing to send a large number of ESPDUs frequently, thus simulators would not usually be able to take advantage of this method for sending their information.

\section{THE JANUS MOVEMENT ALGORITHM}

The Janus family of simulations (which include Janus, Janus-Army, SEES, JCM, and UCCATS) all use essentially the same paradigm for moving entities around the battlefield. There are some differences between the simulations, but the basic content of the algorithms are the same.

Each Janus entity moves in steps. The entity exists at one place for a time, then "jumps" to the next spot, and so on. The amount of each step varies depending on the iype of entity and the terrain, but does not vary with the entity's speed. While the user can set the baseline step size (without terrain constraints factored in) for each type of entity to whatever value is desired, the usual step size chosen for vehicles in many exercises and analyses is fifty meters. A vehicle traveling at $36 \mathrm{~km} / \mathrm{hr}(20 \mathrm{mi} / \mathrm{hr})$ thus travels about $10 \mathrm{~m} / \mathrm{sec}$, and so will "jump" to its next spot every five seconds. 
It would not be too difficult (and would be required for Janus to interoperate via DIS) to add a dead reckoning model to the Janus movement algorithm that would interpolate between jumps, providing continuous movement. The entity's high resolution movement model would still operate in the same "step" fashion, however, so the dead reckoning model would need to be adjusted at almost every step. In the example cited above, that would mean every five seconds. This value would be smaller for faster speeds and/or shorter step lengths. It can be seen immediately that number of ESPDUs per entity issued by Janus-like simulations would be less than that of manned simulators, even though the total number of ESPDUs issued by Janus-like simulations would be far greater than manned simulators due to Janus's modeling of numerous entities. And while Janus, as an event-driven simulation, does not move its entities all at once (but it could-see the discussion below), it can be imagined that situations in which Janus needs to issue a large number of ESPDUs to update the state of a large number of its entities at one time would not be a rare occurrence. If Janus could package up all of these ESPDUs (or shortened forms of them) into one Package PDU, the number of PDUs issued by a Janus simulation could be cut down dramatically.

The SIMNET protocols allowed for a mechanism to place a number of PDUs into a single ethernet frame. That method operated at the link layer in the network protocol hierarchy, not at the application layer, thus did not allow for as much compression or control as the scheme presented below.

\section{PACKAGING}

A simulation may control from ten to hundreds to potentially thousands of entities. As these entities move together over the terrain, they will encounter many terrain features that will modify their movement characteristics (such as their speed) and will require the issuance of an ESPDU. Since many of these entities will be traveling together (in formation) or if there are simply a large number of entities in the simulation, the simulation will need to transmit large numbers of ESPDUs. Similarly, since all entities must "check in" using an ESPDU at least every five seconds, even non-moving entities in a simulation must issue ESPDUs every five seconds.

The ESPDU contains an enormous quantity of information, most of it irrelevant to the routine movement of an entity. Generally, an entity need only say who it is, where it is, how fast it is going, and in which direction. This is especially true of entities in a Janus-like simulation which do not have articulated parts. Similarly, the ESPDU describes the complete state of the entity, when only state change information is really necessary. Therefore, to save network band- 
width, what simulations really need is a Package PDU which would contain all of the relevant header information, and a number of small Entity Update PDUs, which would give state change information about a given entity. A proposed Package PDJ is shown in Table 1.

Table 1: Package PDU

\begin{tabular}{|c|c|c|}
\hline $\begin{array}{c}\text { Field Size } \\
\text { (bits) }\end{array}$ & Data type & PDU Fields \\
\hline 8 & unsigned integer & Protocol version \\
\hline 8 & unsigned integer & Exercise Identifier \\
\hline 8 & enumeration & PDU type (Package PDU =129) \\
\hline 8 & unsigned integer & PDU length \\
\hline 16 & unsigned integer & Entity ID -Site \\
\hline 16 & unsigned integer & Entity ID-Host \\
\hline 32 & unsigned integer & Time stamp \\
\hline 16 & unsigned integer & Package number \\
\hline 16 & unsigned integer & Number of packaged PDUs \\
\hline $\mathrm{n}$ & PDU type $\mathrm{N}$ & PDU \#1 \\
\hline $\mathrm{m}$ & PDU type M & PDU \#2 \\
\hline 0 & PDU type O & PDU \#3 \\
\hline$\ldots$ & $\ldots$ & $\ldots$ \\
\hline \multicolumn{3}{|c|}{ Total =16 bytes (128 bits) plus length of packaged PDUs } \\
\hline
\end{tabular}

The first four bytes represent the PDU header. The next four bytes represent the site and host portions of the Entity ID, so that only the entity portion need be placed in the Entity Update PDU. The next four bytes are the time stamp. According to the DIS standard, the time stamp is twice the number of ticks since the beginning of the current hour (where an hour is divided into $2^{31}$ ticks--approximately $1.676 \mu \mathrm{s} /$ tick) plus 1 if the host's time is synchronized to Universal Coordinated Time. Each of the PDUs in the package needs to specify its time relative to this value (however, it may be necessary to reduce the precision to save space). After the time stamp, the next two bytes give a Package number which would increase by one for each package issue by a host. Missing packages could be detected if there was an interruption in the sequence of package numbers at a receiving host. The next two bytes tell how many PDUs are in the package. Nnte that the length of the enclosed PDUs are not specified. The receiver of this PDU will know how long each enclosed PDU type is. The length of the package PDU is 16 bytes plus the length of the enclosed PDUs. There is no requirement that special PDUs be used in the Package PDU-any PDU or combination of PDUs can be included. 
It is instructive to look at an example using a common type of network, ethernet. A single ethernet frame contains more than 1200 data bytes. Therefore, a Package PDU of roughly this length would minimize the network transmission overhead involved with sending PDUs, and maximize the throughput on an ethernet. An ethernet frame of this length containing one Package PDU could hold eight ESPDUs, up to 120 or 240 of the Entity Update PDUs described below (depending on which format is used), or any conceivable combination. Package PDUs larger than the ethernet maximum frame size would not bring additional advantage. Of course, these values would be different if other network technologies are used, such as Fiber Distributed Data Interconnect (FDDI), Switched Multimegabit Data Service (SMDS), or Asynchronous Transfer Mode (ATM).

\section{ENTITY UPDATE}

The critical information in the ESPDU is (in order of importance) the entity's position, velocity, the time associated with these values, the entity's appearance, as well as any articulated part movements. These are the quantities, which if they change, necessitate the issuance of the ESPDU. The key to data compressicn is to realize that there are some (fairly large) errors which may be made in some of these values which will not make any visual or functional difference in the simulation. Presented here are two different Entity Update PDUs, with different compression and accuracy, that could be used depending on the circumstances to transmit entity state change information. They are called: The Entity Update PDU-the Long Form and The Entity Update PDU-the Short Form. Another possible Entity Update PDU (the EZ Form) is discussed in Appendix A.

The most accurate Entity Update PDU is the Long Form, given in Table 2.

Table 2: Entity Update PDU-the Long Form

\begin{tabular}{|c|c|c|}
\hline $\begin{array}{c}\text { Field Size } \\
\text { (bits) }\end{array}$ & Data type & PDU Fields \\
\hline 8 & unsigned integer & PDU type (EUPDU-LF = 130) \\
\hline 8 & unsigned integer & 8-bit BAM entity orientation-Psi \\
\hline 8 & unsigned integer & 8-bit BAM entity orientation-Theta \\
\hline 8 & unsigned integer & 8-bit BAM entity orientation-Phi \\
\hline 16 & unsigned integer & Entity ID \\
\hline 16 & unsigned integer & Time delta (in units of 2 ticks) \\
\hline 32 & 32-bit floating point & Change in Location - x (meters) \\
\hline 32 & 32-bit floating point & Change in Location - y (meters) \\
\hline 32 & 32-bit floating point & Change in Location - $\mathrm{z}$ (meters) \\
\hline 32 & 32-bit floating point & Linear Velocity - $\mathrm{V}_{\mathrm{x}}$ (m/sec) \\
\hline
\end{tabular}




\begin{tabular}{|l|c|c|}
\hline 32 & 32-bit floating point & Linear Velocity $-\mathrm{V}_{\mathrm{y}}(\mathrm{m} / \mathrm{sec})$ \\
\hline 32 & 32-bit floating point & Linear Velocity $-\mathrm{V}_{\mathrm{z}}(\mathrm{m} / \mathrm{sec})$ \\
\hline \multicolumn{2}{|c|}{ Total $=32$ bytes $(256$ bits $)$} \\
\hline
\end{tabular}

The PDU type and Entity ID fields are self-explanatory. The entity orientation is specified by three angles defined in the DIS standard called psi, theta, and phi. In the DIS 1.0 standard ESPDU, these angles are measured in Binary Angle Measurement (BAM), basically, 360 degrees divided by $2^{32}$. In the DIS 2.0 standard, the angles are measured in radians. In the Entity Update PDU, 8-bit (1.4 degree) accuracy is used, and BAMs are again employed. The time delta is the difference in time between the time stamp in the Package PDU header and the time that this enclosed PDU's information refers to. Since the time delta should be capable of measuring times up to 100-200 milliseconds, and since there are only 16 bits to represent this time, the time delta is measured in units of two ticks (each tick is 1.676 microseconds). Thus the time delta will be capable of representing up to 219.7 milliseconds with an accuracy of 3.35 microseconds. The entity location is a difference measure in meters, accurate to one micron. It represents the difference between the location of this entity in the dead reckoning model at the time represented at the time stamp, and the real (high-resolution) location at the same time. The dead reckoning model should be updated by adding this value to the current value at the given time (not subtracting). Velocity is specified directly in the same fashion as the ESPDU. The total length of the EUPDU-LF is 32 bytes. A Package PDU and four EUPDU-LFs (144 bytes) can fit in the space of one ESPDU (144 bytes), giving a compression factor of approximately four to one.

To compress the information any further requires making certain assumptions and accepting less accuracy in the information. The shorter EUPDU is the EUPDU-Short Form shown in Table 3.

Table 3: Entity Update PDU-the Short Form

\begin{tabular}{|c|c|c|}
\hline $\begin{array}{c}\text { Field Size } \\
\text { (bits) }\end{array}$ & Data type & PDU Fields \\
\hline 8 & unsigned integer & PDU type (EUPDU-SF = 131) \\
\hline 8 & unsigned integer & Time delta (in units of 256 ticks) \\
\hline 16 & unsigned integer & Entity ID \\
\hline 8 & fixed point -12.8 to +12.7 & Change in Location $-\mathrm{x}$ (meters) \\
\hline 8 & fixed point -12.8 to +12.7 & Change in Location $-\mathrm{y}$ (meters) \\
\hline 8 & fixed point -12.8 to +12.7 & Change in Location $-\mathrm{z}$ (meters) \\
\hline 8 & signed integer (coded) & Change in speed (coded-see below) \\
\hline \multicolumn{2}{|r|}{ Total $=8$ bytes (64 bits) } \\
\hline
\end{tabular}


In this PDU, the accuracy of the time delta is decreased to 8-bits. The time delta is measured in 256 -tick units ( 858 microseconds). The range represented is still the same (219.7 milliseconds). The location coordinates are represented by three 8-bit fixed point numbers ranging from -12.8 meters to +12.7 meters. Since ESPDUs are issued if the discrepancy between an entity's dead reckoning location and its actual location is greater than a threshold value, and since the threshold value is generally thought to be in the range of a few meters, this type of fixed point number can represent all necessary values with decimeter resolution, which should be accurate enough for the purposes of the simulation. The final value included is the speed (not the velocity). If the value in this field is called $a$, then the new speed is calculated using the following formula:

New Speed $=$ Old Speed $*(1+$ float $(a) / 512.0)$

This technique allows for changes in the entity's speed of $\pm 25 \%$ with an accuracy of one part in 512. If greater accuracy is needed in a more limited range, the 512.0 value in the equation could be increased. Note that the velocity and orientation cannot be changed by this PDU. To change these values, the Long Form is required. A Packige PDU and 16 of these EUPDU-SFs (144 bytes) could fit in the space of one ESPDU (144 bytes), giving a compression factor of 16 to one.

\section{OTHER PDUS}

The information in a Fire PDU (FPDU) can be compressed and packaged as well. Table 4 shows a Simple Fire PDU.

Table 4: Simple Fire PDU

\begin{tabular}{|c|c|c|}
\hline $\begin{array}{c}\text { Field Size } \\
\text { (bits) }\end{array}$ & Data type & PDU Fields \\
\hline 8 & unsigned integer & PDU type (SFPDU = 133) \\
\hline 8 & unsigned integer & Time delta (in units of 256 ticks) \\
\hline 16 & unsigned integer & Firing Entity ID \\
\hline 16 & unsigned integer & Target Site ID \\
\hline 16 & unsigned integer & Target Host ID \\
\hline 16 & unsigned integer & Target Entity ID \\
\hline 16 & unsigned integer & Event ID \\
\hline 32 & floating point & Munition Speed (m/sec) \\
\hline \multicolumn{2}{|c|}{ Total $=16$ bytes (128 bits) } \\
\hline
\end{tabular}

Most of the fields are self-explanatory. The Event ID is a unique identifier for this fire event. This PDU is only useful for direct fire non-guided non- 
visualized shots (such as a tank gun, or rifle) in which a shooter-target pairing is specified. Shots for direct fire suppression or artillery need to use the Fire PDU. A Package PDU and four SFPDUs ( 80 bytes) can easily fit into the space of one Fire $\mathrm{PDU}$ (84 bytes), giving a compression factor of better than four to one.

Similarly the Detonation PDU can be shortened. A Simple Detonation PDU is shown in Table 5 .

Table 5: Simple Detonation PDU

\begin{tabular}{|c|c|c|}
\hline $\begin{array}{c}\text { Field Size } \\
\text { (bits) }\end{array}$ & Data type & PDU Fields \\
\hline 8 & unsigned integer & PDU type (SDPDU = 134) \\
\hline 7 & unused & Reserved for future use \\
\hline 1 & unsigned integer & Detonation result (Hit $=1$, Miss $=0$ ) \\
\hline 16 & unsigned integer & Time delta (in units of 2 ticks) \\
\hline 16 & unsigned integer & Firing Entity ID \\
\hline 16 & unsigned integer & Event ID \\
\hline 16 & $\begin{array}{c}\text { fixed point }-3276.8 \text { to } \\
+3276.7\end{array}$ & $\begin{array}{c}\text { Location of detonation relative to target }-\mathrm{x} \\
\text { (meters) }\end{array}$ \\
\hline 16 & $\begin{array}{c}\text { fixed point 3276.8 to } \\
+3276.7\end{array}$ & $\begin{array}{c}\text { Location of detonation relative to target }-\mathrm{y} \\
\text { (meters) }\end{array}$ \\
\hline 16 & $\begin{array}{r}\text { fixed point 3276.8 to } \\
+3276.7\end{array}$ & $\begin{array}{c}\text { Location of detonation relative to target }-\mathrm{z} \\
\text { (meters) }\end{array}$ \\
\hline 16 & list of enumerations & Short Munition Record \\
\hline
\end{tabular}

Note that no target is specified, yet the location of the detonation is specified relative to the target using a 16-bit fixed point notation. It is important to remember that the Event ID ties the shot (from the Fire PDU) to the target, thus the target ID can be omitted in the shortened Detonation PDU. Each host must keep track of all active events, so that the target can be obtained from the Event ID. Note that detonation locations relative to the target can be specified to within approximately 3000 meters with decimeter accuracy. If a greater miss distance or more accuracy is needed, the normal Detonation PDU should be used. The detonation result in the third field specifies whether the shooter believes the target was hit or not. The other seven bits can be used for other flags defined in the future. The type of munition is specified using one 16-bit enumeration field. The DIS standard specifies 16 bytes ( 128 bits) to describe the type of munition-five 16-bit enumeration fields and six 8-bit enumeration fields. These can be compressed into one 16-bit data item (see Table 6), when it is realized that certain fields are not necessary for 
the types of non-visualized direct fire munitions for which the Simple Detonation PDU should be used.

Table 6: Short Munition Record

\begin{tabular}{|c|c|c|c|}
\hline $\begin{array}{c}\text { Field Size } \\
\text { Short Form } \\
\text { (bits) }\end{array}$ & $\begin{array}{c}\text { Field Size } \\
\text { DIS } 2.0 \\
\text { (bits) }\end{array}$ & Data type & PDU Fields \\
\hline 0 & 8 & enumeration & Entity Kind \\
\hline 2 & 8 & enumeration & Entity Domain \\
\hline 3 & 16 & enumeration & Entity Country \\
\hline 0 & 8 & enumeration & Entity Category \\
\hline 8 & 8 & enumeration & Entity Subcategory \\
\hline 0 & 8 & enumeration & Entity Specific \\
\hline 0 & 8 & enumeration & Entity Extra \\
\hline 2 & 16 & enumeration & Warhead \\
\hline 0 & 16 & enumeration & Fuze \\
\hline 1 & 16 & enumeration & Quantity \\
\hline 0 & 16 & enumeration & Rate \\
\hline & & Total =2 bytes (16 bits) & \\
\hline
\end{tabular}

In the DIS Detonation PDU, the entity kind, for instance, is always munition. It therefore need not be specified in the Short Munition Record. The domain is either anti-personnel, anti-armor, or battlefield support, so two bits will do. The entity country is either US, UK, French, or Soviet. With a thought to the future, three bits are reserved for this field, yielding a total of eight possible countries. The category is always ballistic, so it need not be specified. The subcategory is where the actual munition is specified. Eight bits (256 values) are reserved for this space even though the DIS 2.0 standard only defines a maximum of 30 . Specific and Extra are unused. Only four types of warheads are needed: HE, HEAT, Kinetic, and Other, so two bits are reserved. The fuze is contact-instant only, therefore need not be specified. Quantity need only be single shot or burst, so one bit is sufficient. Rate is irrelevant to the Short Munition purpose, so can be omitted. Thus the DIS 16 byte munition record can be compressed into a 16 bit field of enumerations under certain conditions, yielding a compression ratio of eight to one. Therefore a Package PDU and five Simple Detonation PDUs (96 bytes) can easily fit into the space of one Detonation PDU (100 bytes), giving a compression factor of over five to one.

Finally, what should be sent when a unit is stopping or sitting still? Two types of PDUs are needed, one containing information when a unit has just 
stopped (a Stopped PDU or SPDU) and another saying that the unit still exists and continues to be stationary. First, the SPDU (which is very similar to a EUPDU-SF) is shown in Table 7.

Table 7: Stopped PDU

\begin{tabular}{|c|c|c|}
\hline $\begin{array}{c}\text { Field Size } \\
\text { (bits) }\end{array}$ & Data type & PDU Fields \\
\hline 8 & unsigned integer & PDU type (SPDU = 135) \\
\hline 8 & unsigned integer & Time delta (in units of 256 ticks) \\
\hline 16 & unsigned integer & Entity ID \\
\hline 8 & fixed point -12.8 to +12.7 & Change in Location - x (meters) \\
\hline 8 & fixed point -12.8 to +12.7 & Change in Location - y (meters) \\
\hline 8 & fixed point -12.8 to +12.7 & Change in Location - (meters) \\
\hline 8 & unsigned integer & 8-bit BAM entity orientation \\
\hline \multicolumn{2}{|r|}{ Total $=8$ bytes (64 bits) } \\
\hline
\end{tabular}

All entries are of the same form as in EUPDU-SF except the last field. In the EUPDU-SF, the last field represented a speed; here it represents an orientation of the entity with respect to a vertical axis perpendicular to the ground, East being zero and increasing positive counterclockwise. A Package PDU and 16 Stopped PDUs (144 bytes) can fit in the same space as one ESPDU (144 bytes) giving a compression factor of 16 to one.

When an entity continues to be stationary it can issue an Existence PDU, shown in Table 8.

Table 8: Existence PDU

\begin{tabular}{|c|c|c|}
\hline $\begin{array}{c}\text { Field Size } \\
\text { (bits) }\end{array}$ & Data type & PDU Fields \\
\hline 8 & unsigned integer & PDU type (EPDU =136) \\
\hline 8 & unused & padding \\
\hline 16 & unsigned integer & Entity ID \\
\hline \multicolumn{2}{|r|}{ Total $=4$ bytes (32 bits) } \\
\hline
\end{tabular}

A Package PDU and 32 Existence PDUs (144 bytes) can fit in the same space as one ESPDU (144 bytes) giving a compression factor of 32 to one.

Yet this PDU may not be entirely necessary. Why is it necessary to confirm the existence of every entity every five seconds? Perhaps what is needed is a Host Existence PDU, independent of the Package PDU, which tells all of the other hosts on the network that the sending host's entities are all still in the game. This PDU 
would be issued every five seconds instead of the 100 to 200 Existence PDUs. The Host Existence PDU (HEPDU) is shown in Table 9.

Table 9: Host Existence PDU

\begin{tabular}{|c|c|c|}
\hline $\begin{array}{c}\text { Field Size } \\
\text { (bits) }\end{array}$ & Data type & PDU Fields \\
\hline 8 & unsigned integer & Protocol version \\
\hline 8 & unsigned integer & Exercise Identifier \\
\hline 8 & enumeration & PDU type (HEPDU $=137$ ) \\
\hline 8 & unsigned integer & PDU length \\
\hline 16 & unsigned integer & Site \\
\hline 16 & unsigned integer & Host \\
\hline 32 & unsigned integer & Time stamp \\
\hline \multicolumn{2}{|r|}{ Total $=12$ bytes $(96$ bits) } \\
\hline
\end{tabular}

The fields are all the same as in the Package PDU. Since the compression factor in this PDU's case is dependent on how many entities are controlled by the host, a general number cannot be assigned. But assuming 100 entities all issuing ESPDUs (14,400 bytes), the compression factor is 1200 to one. Assuming 100 entities all issuing Existence PDUs in a Package PDU (416 bytes), the compression factor is 35 to one.

\section{Discussion}

It is clear that the DIS Entity State PDU is not designed for a large-scale bandwidth-limited real-time distributed simulation. For instance, the country of an entity is specified in every ESPDU. Why is this necessary? Similarly, if an entity starts the battle as an M1A1 tank, the probability that it will stay an M1A1 throughout the battle is pretty close to unity. Why include redundant information in the most crucial network-overloading PDU? Generally it is thought that this redundant information will enable simulators to keep track of only those entities on the battlefield that are of interest, with the list of interesting entities changing dynamically throughout the battle. However, with today's large random access memory computers, this capability becomes less important. One need only ensure that occasionally each entity sends out a full ESPDU to enable other simulations to know about the entity if they are just joining or are rejoining the simulation. Exactly how often this needs to be done is a matter for further research. What is needed is occasional large packages of information which require only one interrupt to be read, but contain as much information as the dozens of normal PDUs. 
The type of compression PDUs described in the above sections are not intended to be complete or the final word. Experiments should be performed with a real distributed simulation to see what changes need to be made to perfect the design. Perhaps some types would not be used very much and should be dropped. For instance, there are two different Entity Update PDUs. Which would be used the most?

The following describes the algorithm to determine which EUPDU to send and how they are sent.

- When an entity's dead reckoning model differs by a certain amount from its high resolution movement model, other network nodes need to be informed. The node controlling the entity to be updated calculates the amount that the entity's position, velocity, and orientation need to be changed to bring the dead reckoning algorithm back into line with the high resolution model. The node evaluates each of the three types of PDUs (the ESPDU and the two EUPDUs) to see how well each performs the update task relative to some standard (i.e. the position can't be off by a certain amount, the velocity can't be off by another specified amount, etc.) The smallest PDU which meets the criteria is selected to send the information.

- If a Package PDU already exists, the new EUPDU is put in the package, with a current time delta relative to the package's time stamp.

- If a Package PDU does not exist, one is created, and header information is loaded into it along with the Update PDU. The Update PDU time delta will be zero since it was created at the same time as the Package PDU. A clock is started now which will count down the time to send the Package. Packages can be held for a reasonably long time (perhaps 100 milliseconds) before their information begins to get stale. The actual maximum shelf time for a Package will need to be determined by performing an analysis using a distributed simulation.

- PDUs continue to collect in the Package (including any Stopped PDUs, Simple Fire PDUs, Simple Detonation PDUs, etc.) until the Package shelf time has expired. Then the Package is sent multicast onto the network, the sending node updates its own dead reckoning model with the new information, and the process begins again.

- When a node receives a Package, it unpacks it, and updates the dead reckoning models of the described entities using the various time stamps to calibrate the model. For instance, if entity $A$ was at $(x, y, z)$ at time $t$, but the 
current time is $t+\Delta t$, the entity's dead reckoning model is adjusted such that entity $A$ would have been at the specified position at time $t$. This process of "backdating" ensures that the dead reckoning model at the receiving node exactly matches the dead reckoning model at the transmitting node. This process requires that the computers on the network keep exact time by being coordinated with Universal Coordinated Time. Of course, since only approximate information is sent in the smaller EUPDUs, the dead reckoning model will not exactly match the high resolution model. This is acceptable, however, because it will be true of the sending host's dead reckoning model as well.

The last point requires an elaboration. Exact data is not being sent in the small EUPDUs to save space. But this does not have to be the case. What if the movement of all entities was constrained to the vertices of a grid with edges of a decimeter? Then the smaller PDUs would be transmitting exact information, and the small PDUs would be identical in resolution to the full ESPDU. If all entities' high resolution models were constrained to a decimeter grid, but the dead reckoning models were not, then the entities would not even be seen to move in a "jerky" fashion as the dead reckoning model would smooth the motion out (and the dead reckoning models would be in error by a maximum of $\pm 5 \mathrm{~cm}$ ). Or the dead reckoning models could be quantized as well. It seems reasonable that no militarilysignificant effect can occur, even if the entity is fighting in a building, if an entity must take up a position $5 \mathrm{~cm}$ to the right or left of where it "really wants" to be, as long as everyone else knows that is where it is. This "quantization" of the motion of entities is not particularly different than what really occurs with floating point (which quantizes space with a seven digit accuracy) or with double precision (17 digit or $10^{-10}$ meter accuracy). If entities' positions were described by 32 -bit integers and measured in decimeters, the position values could range up to $\pm 200,000$ kilometers, which would encompass most of the space from the Earth to the Moon. Going to integer space coordinates would thus have the advantage of being able to send exact Entity Updates in very little space, without sacrificing anything important.

Finally, it is important to discuss the Janus movement algorithm, and how changes could be made to it to help save bandwidth and time. Janus is not a realtime simulation; it is an event-driven simulation. But it could be made into a pseudo-real-time simulation without much effort. Instead of updating a unit's movement depending on a spatial distance, Janus could update the movement of every entity depending on an elapsed time. For example, instead of moving each unit in 50 meter jumps, Janus could update every entity's position every so many units of time. To operate in real-time, Janus would have to anticipate a little to be 
able to perform the calculations without having the information get stale. For instance, if Janus updates every unit's position every second, then to update everyone at $t=10$ seconds, the Janus movement process would wake up at $t=9.9$ seconds, do all of the movement calculations, decide about which PDUs need to be sent, and Package them all up for transmittal at $t=10$ seconds. Any other PDUs (fire, detonation, etc.) which need to be sent in this time frame would be included in the Package as well. The amount of time necessary to do all of the movement calculations is the key to this entire scheme. If it can be kept low (below 100 milliseconds) then one could imagine just sending one Package (containing every PDU that needs to be sent in that time frame) every 100 milliseconds or so. Then all of the information necessary to run a Janus simulation with hundreds of entities could be sent with just 10 PDUs per second.

Does this make any sense? If a simulation controls 200 entities, it might under very unlikely circumstances need to update the position of $40 \%$ of its entities every tenth of a second. Under this assumption, it might need to issue 70 EUPDUSFs, ten EUPDU-LFs, and ten simple fire or detonation PDUs, so the size of the Package PDU would be $560+320+160+16$ (for the package) $=1056$ bytes. If ethernet is chosen as the physical network medium, a maximum of roughly 40 Janus hosts could issue this amount of data ten times per second. Thus the total number of entities that could exist on the ethernet would be 8000 . If Janus needed to issue 80 ESPDUs and ten Fire or Detonation PDUs every 100 milliseconds (11520 $+1000=12520$ bytes) and ethernet was the network medium, a maximum of four Janus hosts (of 200 entities each: total 800 entities) could exist on the network. Of course the actual amount of traffic is likely to be much smaller than in this example, and thus it is likely that more entities could exist under both the uncompressed and compressed cases. Nevertheless, if the compression described in this example could be achieved with regularity, the result of compression would be a factor of ten increase in the battle space.

\section{CONCLUSION}

Data compression is not only possible between distributed simulations br it is tailor-made for such simulations which tend to require the transmission of numerous Entity State PDUs for many different entities very frequently. Eight new PDUs have been described which yield compression factors from three to one up to 1200 to one. Sustained compression at this rate could increase by an order of magnitude the number of possible entities in a distributed simulation using standard network media. Tests need to be performed on an actual distributed simulation to determine the usefulness of these proposed PDUs and to suggest any additions or improvements to the list. 


\section{APPENDIX A: AN EVEN SHORTER EUPDU}

Even greater compression of the entity state information can be achieved if everything but the location is removed, and even less accuracy is accepted. An EUPDU consistent with this idea is the EZ form, shown in Table 10.

Table 10: Entity Update PDU-EZ

\begin{tabular}{|c|c|c|}
\hline $\begin{array}{c}\text { Field Size } \\
\text { (bits) }\end{array}$ & Data type & PDU Fields \\
\hline 8 & unsigned integer & PDU type (EUPDU-EZ =132) \\
\hline 8 & unsigned integer & Entity ID \\
\hline 5 & fixed point -1.6 to +1.5 & Change in Location $-\mathrm{x}$ (meters) \\
\hline 5 & fixed point -1.6 to +1.5 & Change in Location $-\mathrm{y}$ (meters) \\
\hline 5 & fixed point -1.6 to +1.5 & Change in Location $-\mathrm{z}$ (meters) \\
\hline 1 & unsigned integer & Time stamp (add 50 msec or not) \\
\hline \multicolumn{2}{|r|}{ Total $=4$ bytes (32 bits) } \\
\hline
\end{tabular}

The first item to note about the EZ PDU is that the Entity ID is only eight bits long rather than the 16 bits specified by the DIS standard. This means that this PDU can only be used by hosts controlling 256 entities or less. This is not a terribly restrictive requirement, if each host is planned to be a small, cheap, PC-type node which would only model 200 or so entities. The location deltas are specified by 5bit fixed point numbers representing -1.6 meters to +1.5 meters, with decimeter accuracy. The "time stamp" has been reduced to one bit, representing whether to add a fixed amount to the Package PDU time stamp or not (in this case 50 milliseconds). Fifty milliseconds is simply a guess as to what a likely addition might be. Further study under actual simulation conditions are required to determine what the best value for this number should be on a permanent basis. One Package PDU and 32 of these EUPDU-EZs (144 bytes) can fit in the space of one ESPDU, giving a compression factor of 32 to one.

No change in velocity or orientation is specified in this PDU. Nevertheless, it could be very useful in situations where slight discrepancies exist between the dead reckoning model of a unit and its high-resolution movement model that do not merit any more detail. Of course since this PDU cannot correct the dead reckoning model with exact accuracy (except when position is quantized), these PDUs will need to be issued more often than would a true ESPDU; however, the exact difference in issuance rate needs to be determined under actual simulation conditions to judge what is required and what is not with regard to accuracy. 


\section{APPENDIX B: THE DIS 2.0 ENTITY STATE PDU}

For the reader's information, the content of the DIS Entity State PDU (ESPDU) is listed here in Table 10. This information is from the March 1993 version of the DIS 2.0 Draft Standard Document.

Table 10: DIS Entity State PDU

\begin{tabular}{|c|c|c|}
\hline $\begin{array}{c}\text { Field Size } \\
\text { (bits) }\end{array}$ & Data type & PDU Fields \\
\hline 8 & unsigned integer & Protocol version \\
\hline 8 & unsigned integer & Exercise Identifier \\
\hline 8 & enumeration & PDU type \\
\hline 8 & unsigned integer & PDU length (in 32-bit words) \\
\hline 16 & unsigned integer & Entity ID $\rightarrow$ Site \\
\hline 16 & unsigned integer & Entity ID-Host \\
\hline 16 & unsigned integer & Entity ID-Entity \\
\hline 8 & unused & Padding \\
\hline 8 & unsigned integer & Force ID \\
\hline 8 & enumeration & Entity Type-Entity Kind \\
\hline 8 & enumeration & Entity Type-Domain \\
\hline 16 & enumeration & Entity Type-Country \\
\hline 8 & enumeration & Entity Type-Category \\
\hline 8 & enumeration & Entity Type-Subcategory \\
\hline 8 & enumeration & Entity Type-Specific \\
\hline 8 & enumeration & Entity Type-Extra \\
\hline 8 & enumeration & Guise Entity Type-Entity Kind \\
\hline 8 & enumeration & Guise Entity Type-Domain \\
\hline 16 & enumeration & Guise Entity Type-Country \\
\hline 8 & enumeration & Guise Entity Type-Category \\
\hline 8 & enumeration & Guise Entity Type-Subcategory \\
\hline 8 & enumeration & Guise Entity Type-Specific \\
\hline 8 & enumeration & Guise Entity Type-Extra \\
\hline 32 & unsigned integer & Time stamp $(2 *$ ticks + flag $)$ \\
\hline 64 & double precision float & Entity Location - $\mathrm{x}$ (meters) \\
\hline 64 & double precision float & Entity Location - y (meters) \\
\hline 64 & double precision float & Entity Location $-\mathrm{z}$ (meters) \\
\hline 32 & floating point & Entity Linear Velocity $-\mathrm{x}(\mathrm{m} / \mathrm{sec})$ \\
\hline 32 & floating point & Entity Linear Velocity - y $(\mathrm{m} / \mathrm{sec})$ \\
\hline 32 & floating point & Entity Linear Velocity $-\mathrm{z}(\mathrm{m} / \mathrm{sec})$ \\
\hline
\end{tabular}


Data Compression Enhancements to the DIS Protocol...

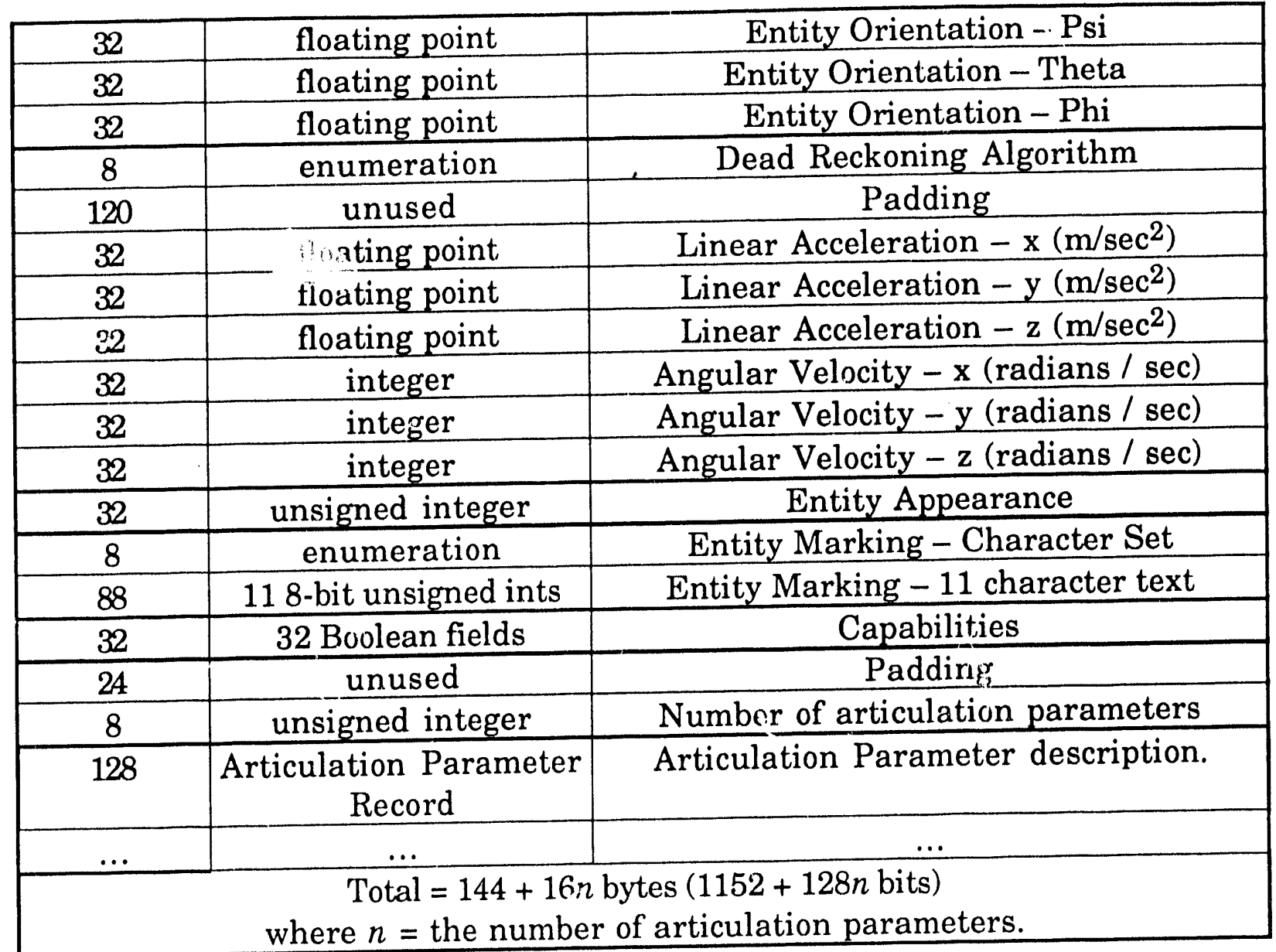

\section{REFERENCES}

SIMNET: Advanced Technology for the Mastery of Warfighting, BBN Laboratories Report No. 6787.

Military Standard-Protocol Data Units for Entity Information and Entity Interaction in a Distributed Interactive Simulation-Draft Version 2.0, IST-CR-9301, September 4, 1993.

0.1 Compression and DIS Communications, Unpublished Fax from Loral Defense Systems Krona, distributed at the DIS Communications Architecture and Security Subgroup Meeting, Armstrong Labs, January 1993, dated DEC 04, 1992, 08:45AM, p.16-18 

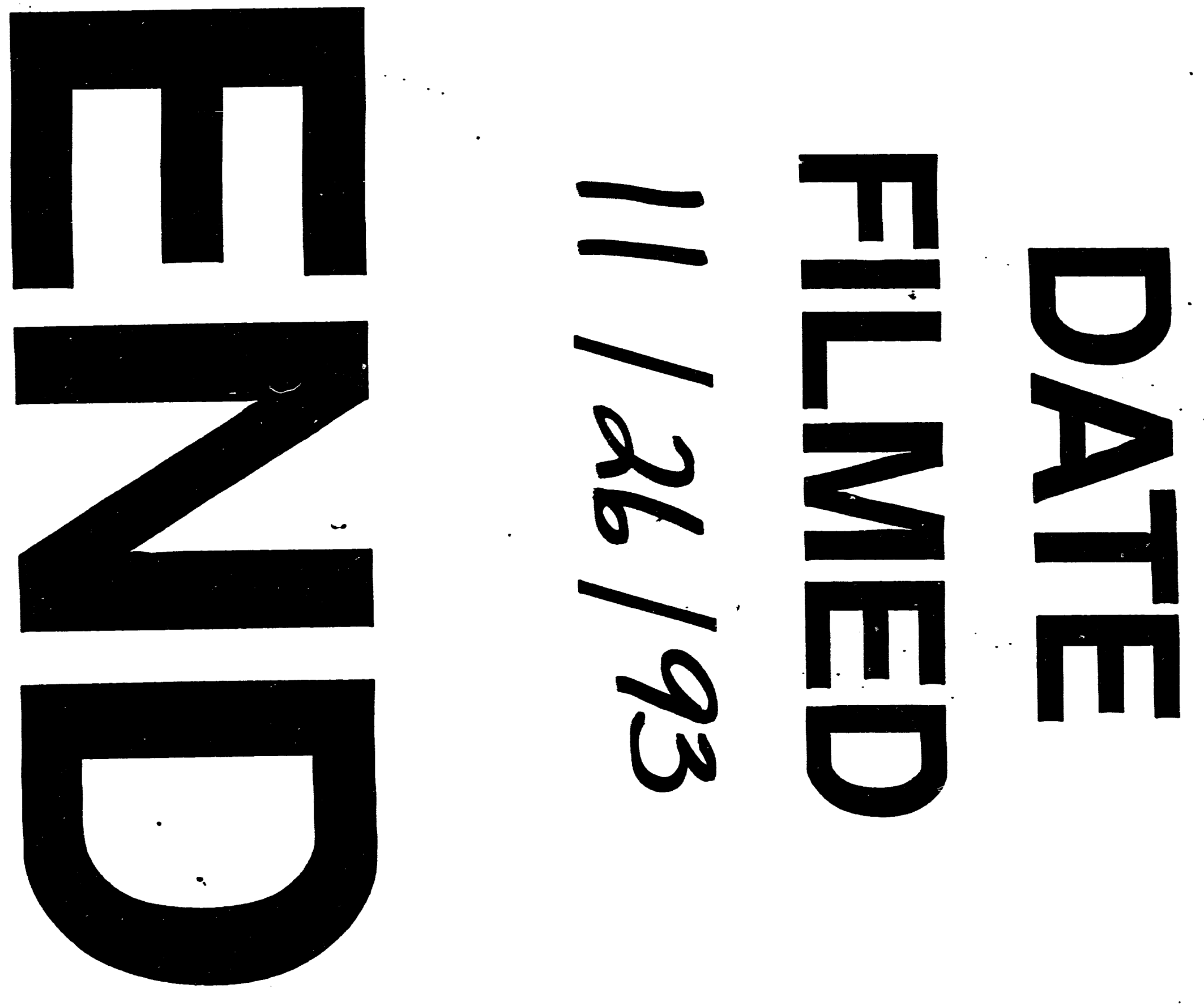
